\section{Estudo \\ cabebate}

em Testão

Plamejamento
Revista Estudo \& Debate, Lajeado, v. 25, n. 3, 2018. ISSN 1983-036X

DOI: http://dx.doi.org/10.22410/issn.1983-036X.v25i3a2018.1858

\title{
DESCRIÇÃO MORFOLÓGICA DOS GRÃOS DE PÓLEN DOS CAMPOS E FLORESTAS DO MUNICÍPIO DE ARVOREZINHA, PLANALTO DO RIO GRANDE DO SUL, BRASIL
}

\author{
Paulo Eduardo Liskoski ${ }^{1}$, Andreia Cardoso Pacheco Evaldt ${ }^{2}$, Jefferson Nunes Radaeski ${ }^{3}$, \\ Soraia Girardi Bauermann ${ }^{4}$, Mariela Inês Secchi ${ }^{5}$
}

\begin{abstract}
Resumo: A vegetação da regiáo do planalto do Rio Grande do Sul é caracterizada por um mosaico de campos e floresta de araucária. É apresentada a morfologia dos grãos de pólen de 28 espécies de 14 famílias (Piperaceae, Iridaceae, Commelinaceae, Bromeliaceae, Cyperaceae, Poaceae, Rosaceae, Begoniaceae, Myrtaceae, Melastomataceae, Malvaceae, Rubiaceae, Solanaceae, Asteraceae), coletadas nos campos e florestas do município de Arvorezinha do estado do Rio Grande do Sul, com a finalidade de registrar a diversidade polínica do planalto sul-riograndense. Foi realizado tratamento acetolítico e montadas cinco lâminas em gelatina glicerinada para cada espécie. Os grãos de pólen foram descritos quanto sua unidade polínica, ao tamanho, ao âmbito do pólen, a forma, ao número de aberturas e a ornamentação. Espécies já descritas anteriormente tiveram seus dados confrontados para observaçôes de possíveis variaçôes no tipo polínico. Dezesseis descriçôes polínicas são inéditas para o estado, aprofundando o conhecimento da diversidade polínica do estado do Rio Grande do Sul. O registro da diversidade polínica regional apresentada pode ser utilizado para identificação de grãos de pólen dispersos em sedimentos quaternários. Os dados propiciam análises de reconstrução da vegetaçáo que indicam espécies constituintes da vegetação natural. Informaçôes sobre a flora original a partir de seus respectivos grãos de pólen são cruciais para elaboração de estratégias de manejo de áreas degradadas.
\end{abstract}

Palavras-chave: Palinologia. Palinomorfos. Vegetação. Mata Atlântica.

1 Laboratório de Palinologia, Universidade Luterana do Brasil. pauloliskoski@gmail.com

2 Laboratório de Palinologia, Universidade Luterana do Brasil. andreia.biologia@gmail.com

3 Laboratório de Palinologia, Universidade Luterana do Brasil. jefferson.radaeski@gmail.com

4 Laboratório de Palinologia, Universidade Luterana do Brasil. soraia.bauermann@ulbra.br

5 Setor de Botânica e Paleobotânica do Museu de Ciências Naturais da Univates, Programa de Pós-Graduação em Ambiente e Desenvolvimento, Univates. maries@universo.univates.br 


\title{
DESCRIPTION OF THE POLLEN GRAINS FROM GRASSLANDS AND FORESTS OF THE ARVOREZINHA REGION, PLATEAU OF RIO GRANDE DO SUL, BRAZIL
}

\begin{abstract}
The vegetation of the plateau of Rio Grande do Sul is characterized by a mosaic of grassland and araucaria forest. Pollen morphology of 28 species of 14 families (Piperaceae, Iridaceae, Commelinaceae, Bromeliaceae, Cyperaceae, Poaceae, Rosaceae, Begoniaceae, Myrtaceae, Melastomataceae, Malvaceae, Rubiaceae, Solanaceae, Asteraceae), are presented. The species analyzed occur in the grasslands and forests of Arvorezinha region of Rio Grande do Sul state, Brazil. The aim is to record the pollen diversity of Rio Grande do Sul plateau. The pollen samples were chemically processed by acetolysis treatment and assembled five slides in glycerinated jelly for each species. Pollen grains were described as to their pollen unit, size, amb, shape, number of apertures and ornamentation. Species previously described had their data checked to possible variation in the pollen type. Sixteen pollen descriptions are unpublished for the State, deepening the knowledge of the pollen diversity of the state of Rio Grande do Sul. The regional pollen diversity record presented can be used to identify pollen grains dispersed in Quaternary sediments. The data provide analyzes of the reconstruction of vegetation that indicate species of natural vegetation. Information about the original vegetation from their respective pollen grains is crucial for the elaboration of strategies for the management of degraded environments.
\end{abstract}

Keywords: Palynology. Palynomorph. Vegetation. Atlantic forest.

\section{Introdução}

Os estudos paleoambientais exigem uma precisa identificação dos grupos polínicos fósseis e atuais. Estes estudos palinológicos possibilitam uma comparação entre os ambientes passados com a vegetação atual, possibilitando o entendimento e interpretação das mudanças da vegetação (Evaldt et al. 2009).

A elaboração de um banco de dados de grãos de pólen de espécies ocorrentes na região poderá ser utilizada como ponto de partida para a reconstituição do cenário paleoambiental e definir a flora nativa do passado. Conhecer a vegetação natural permitirá um manejo adequado para a recuperação de áreas degradadas e auxiliar a estabelecer as rotas migratórias das espécies. Além disso, os grãos de pólen também apontam para perturbaçóes na flora através da identificação de pólen exótico.

Os avanços na caracterização de morfologias de grãos de pólen modernos promovem progressos na identificação de pólen contido em amostras polínicas. Neste sentido, na última década diversos esforços foram destinados à descrição polínica da flora sul brasileira (EVALDT et al., 2009; BAUERMANN et al., 2013; RADAESKI et al., 2014; REDE DE CATÁLOGOS POLÍNICOS ONLINE, 2017). Resultados satisfatórios sobre a aplicação de conhecimento de pólen atual para identificação de táxons em amostras palinológicas do quaternário já foram evidenciados no sul do Brasil. Por exemplo, táxons da flora de Savana Estépica Parque do Estado tiveram seus grãos de pólen descritos (Radaeski et AL. 2014) e posteriormente estes grãos de pólen foram identificados em amostras quaternárias (Evaldt et al. 2014). Com maiores dados sobre a morfologia atual do sul do Brasil, informaçóes ecológicas adicionais podem ser reveladas para interpretação de amostras polínicas de mel (PILOTTO et al., 2016) e fósseis (RADAESKI; CUNHA; BAUERMANN, 2017), entre outros. Contudo, a grande diversidade florística regional no sul do Brasil ainda necessita de descrição de seus respectivos grãos de pólen. Uma destas regióes está localizada no nordeste 
do Rio Grande do Sul, onde somente recentemente foram apresentados dados de pólen atual para as florestas e campos (RADAESKI et al. 2014). Trabalhos de reconstrução e de história ambiental com base em grãos de pólen que estão em desenvolvimento (SECCHI, 2017) somente são possíveis de serem interpretados com base em descriçôes polínicas modernas. Neste sentido, o objetivo deste estudo é apresentar as assembleias polínicas atuais como padrão da vegetação capaz de subsidiar manejo de áreas degradadas.

\section{Material e Métodos}

O município de Arvorezinha está localizado a nordeste do estado do RS, a 200 $\mathrm{km}$ da capital Porto Alegre. Tem uma área de 271, $643 \mathrm{~km}$ (IBGE, 2016), situada nas coordenadas $28^{\circ} 52^{\prime} 23,4^{\prime}$ 'S e $052^{\circ} 09^{\prime} 56,2$ W. Na vegetação do município destacam-se: a Floresta Ombrófila Mista, capoeiras, lavouras e campos limpos, sendo que em poucas áreas encontra-se vegetação nativa (FIGURA 1), destacando-se o pinheiro e a erva-mate, pela sua utilidade. No extrato arbóreo, há predominância de Eugenia uniflora, Uncaria tomentosa e Solanaceae. No extrato herbáceo com proeminência de espécies do gênero Commelina (Commelinaceae), Senecio (Asteraceae) e Cyperus (Cyperaceae). (ADMINISTRAÇÃO MUNICIPAL DE ARVOREZINHA, 2017).

Figura 1: Campo e floresta do planalto sul-rio-grandense, município de Arvorezinha

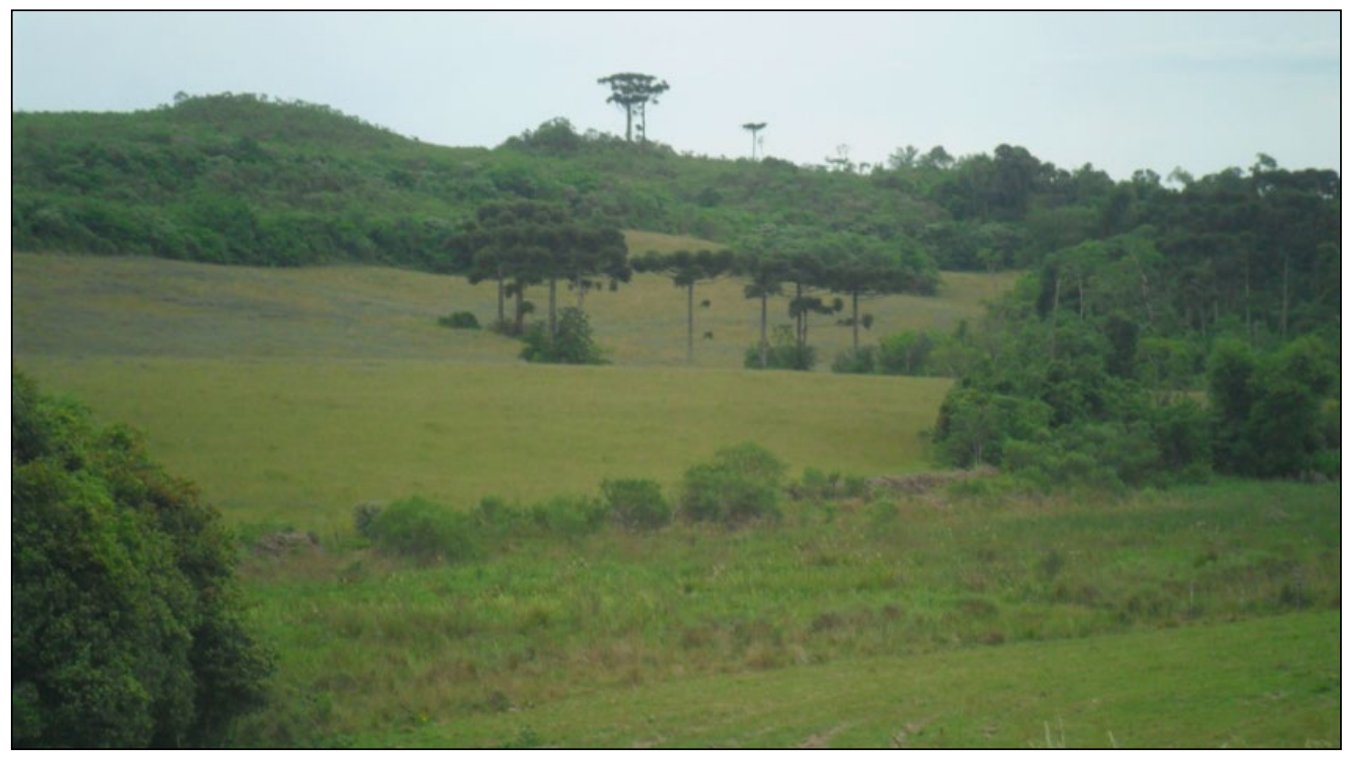

Fonte: Elaboração própria.

As plantas coletadas em três pontos do município (FIGURA 2) através pelo método de caminhamento foram prensadas e desidratadas, posteriormente fixadas, montando-se exsicatas depositadas no Herbário do Museu de Ciências Naturais da Universidade Luterana do Brasil (MCN/HERULBRA). A partir do material herborizado, foram coletadas anteras para tratamento químico acetolítico do material polínico (ERDTMAN, 1952). 
Figura 2: Mapa de arvorezinha delimitando áreas de campos e florestas, assim como a localização dos pontos de coleta.

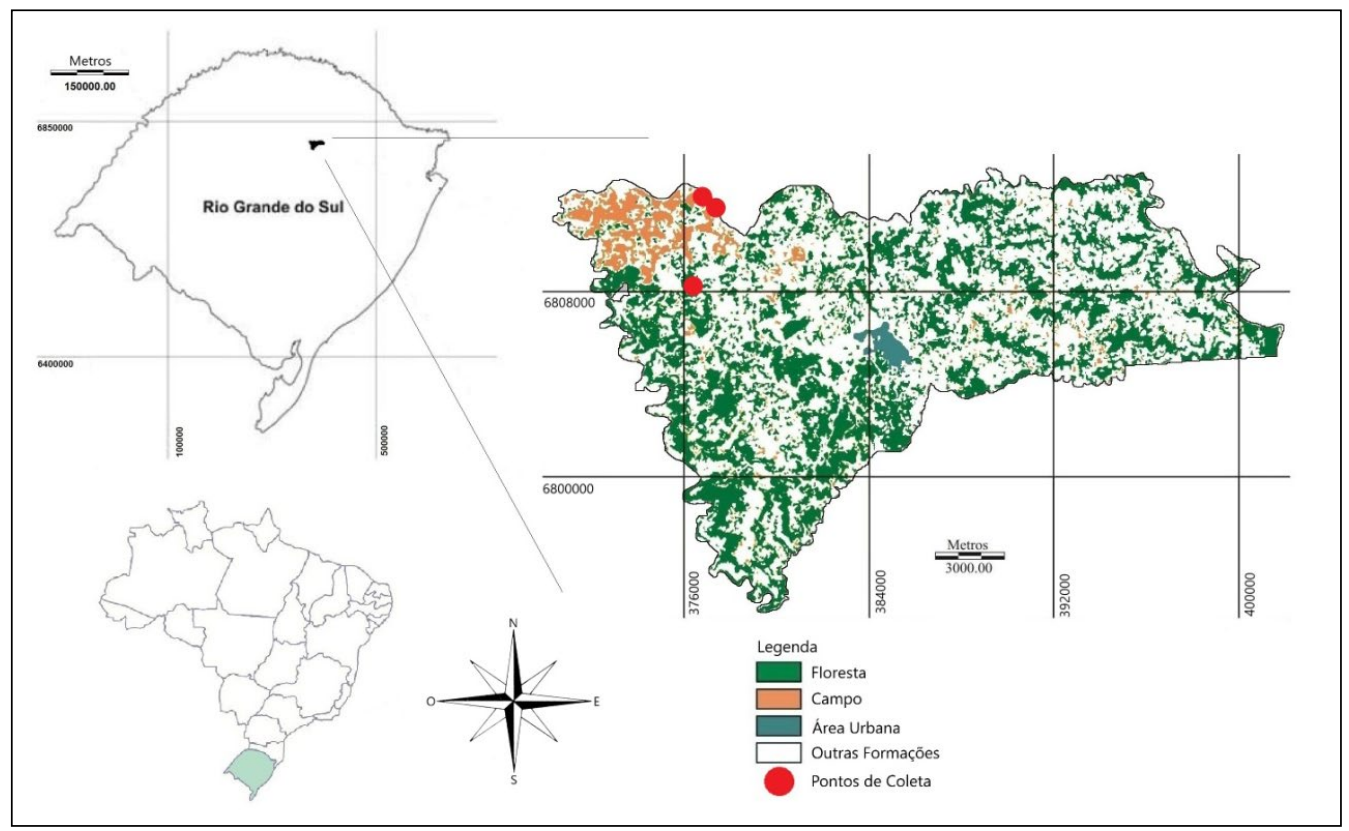

Fonte: CEMIN; PÉRICO; REMPEL (2005); PÉRICO e CEMIN (2006).

Foram preparadas cinco lâminas de cada espécie em gelatina glicerinada e parafina para análise em microscópio óptico (SALGADO-LABOURIAU, 2007). Os grãos de pólen foram descritos quanto sua unidade polínica, ao tamanho, ao âmbito, a forma, ao número de aberturas e a ornamentação. Foram medidos 25 grãos de cada espécie em microscopia óptica com aumento de $1.000 \mathrm{x}$, em vista equatorial, o diâmetro polar (P) e o diâmetro equatorial (E), espessura da exina (Ex) e a ornamentação (Or) se for maior que $1 \mu \mathrm{m}$, todas as mediçóes expressas em micrômetros, seguindo protocolo proposto por Barth e Melhem (1988). Em grãos de pólen de Cyperaceae, seguiu-se o critério de Moar e Wilmshurst (2003). Em grãos de pólen esféricos foi medido apenas o diâmetro (D). Nas espécies monossulcadas foram medidos o diâmetro polar (P), diâmetro equatorial menor (Dem), diâmetro equatorial maior (DEM), devido à tendência de os grãos de pólen cair em vista polar. As descrições estão organizadas em ordem evolutiva conforme APG IV (2016). Os nomes das espécies e autores seguem a Lista de Espécies da Flora do Brasil 2020. Foram realizadas fotografias em vista polar e equatorial dos grãos de pólen. Para as descriçóes polínicas foi utilizada a metodologia e terminologia propostas por Barth e Melhem (1988) atualizada por Punt et al. (2007) e consultada a chave de identificação da rede de catálogos polínicos on-line (RCPol, 2018). 


\section{Resultados e Discussáo}

Foram analisados grãos de pólen de 28 espécies, distribuídas em 26 gêneros pertencendo a 14 famílias (TABELA 1). As espécies estão apresentadas seguindo o sistema de classificação filogenética proposta pelo APG IV.

\section{Piperaceae}

Piper mikanianum (Kunth) Steud.(Figura 3 A-B)

Descrição polínica: Mônade, pequeno, simetria bilateral, isopolar, âmbito circular, prolato, monocolpado, colpo longo, exina escabrada. Sexina e nexina de mesma espessura.

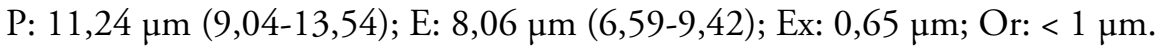

Observaçóes ecológicas: Herbácea, com ocorrência na Amazônia, cerrado e mata atlântica, popularmente conhecida como pariparoba (FLORA DO RIO GRANDE DO SUL, 2018; FLORA DO BRASIL, 2018).

\section{Iridaceae}

Sisyrinchium sp. L. (Figura 1 C-D)

Descrição polínica: Mônade, médio, simetria bilateral, isopolar, âmbito elíptico, prolato-esferoidal, monossulcado, exina microrreticulada. Sexina e nexina de mesma espessura. P: 30 (26-34); DEM: 37,2 $\mu \mathrm{m}$ (33-42); Dem: 26,42 (24-29,5); Ex: 1,42 $\mu \mathrm{m}$.

Referência prévia: Radaeski et al. 2011.

Observaçóes ecológicas: Herbácea, com ocorrência no cerrado, mata atlântica, pampa e pantanal (FLORA DO BRASIL, 2018).

\section{Commelinaceae}

Tripogandra sp. Raf. (Figura 3 E-F)

Descrição polínica: Mônade, médio, simetria bilateral, isopolar, âmbito elíptico, prolato, monossulcado, exina microrreticulada. Sexina e nexina de mesma espessura. P: 19,8 (18-23); DEM: 23,28 $\mu \mathrm{m}$ (21-26); Dem: 13,28 (10-17); Ex: $1 \mu \mathrm{m}$.

Referência prévia: Poole e Hunt, 1980.

Observaçóes ecológicas: Herbácea, com ocorrência na Amazônia, caatinga, cerrado, mata Atlântica, pampa e pantanal (FLORA DO BRASIL, 2018).

\section{Bromeliaceae}

\section{Tillandsia sp. L. (Figura 3 G-H)}

Descrição polínica: Mônade, médio, simetria bilateral, isopolar, âmbito elíptico, oblato-esferoidal, monossulcado, exina reticulada-heterobrocada, medindo até $1,5 \mu \mathrm{m}$ de diâmetro, retículos maiores no mesocolpo e menores no apocolpo. Sexina e nexina de

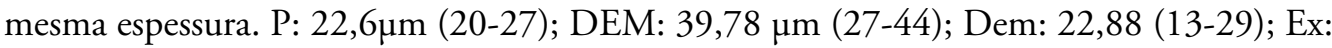
$1,04 \mu \mathrm{m}$.

Referência prévia: Bauermann et al., 2013; Radaeski et al., 2014. 
Observaçóes ecológicas: Herbácea, com ocorrência na Amazônia, caatinga, cerrado, mata atlântica, pampa e pantanal, popularmente conhecida como cravo-do-mato (FLORA DO RIO GRANDE DO SUL, 2018; FLORA DO BRASIL, 2018).

\section{Cyperaceae}

Cyperus surinamensis Rottb. (Figura $3 \mathrm{I}-\mathrm{J}$ )

Descrição polínica: Mônade, médio, simetria bilateral, anisopolar, âmbito subcircular, prolato (em forma de pêra, com maior tamanho em um dos pólos, apresentado em DEM e Dem), inaperturado, apresenta 3 áreas de fragilidade na exina, exina microrreticulada. Sexina e nexina de mesma espessura. P: 35,72 $\mu \mathrm{m}$ (32-41); DEM: 25,54 $\mu \mathrm{m}(22-30,5)$; Dem: 4,4 $\mu \mathrm{m}$ (6-8); Ex: 1,12 $\mu \mathrm{m}$; Orn: $<1 \mu \mathrm{m}$.

Observaçóes ecológicas: Herbácea, com ocorrência na Amazônia, caatinga, cerrado, mata atlântica, pampa e pantanal, popularmente conhecida como tiririca (FLORA DO BRASIL, 2018; ALMEIDA JR et al., 2008).

Fimbristylis autumnalis (L.) Roem. \& Schult. (Figura 3 K-L)

Descrição polínica: Mônade, médio, simetria bilateral, anisopolar, âmbito subcircular, subprolato (em forma de pêra, com maior tamanho em um dos pólos, apresentado em DEM e Dem), inaperturado, apresenta áreas de fragilidade na exina, exina microrreticulada. Sexina e nexina de mesma espessura. P: 31,62 $\mu \mathrm{m}$ (26-36); DEM: 25,16 $\mu \mathrm{m}$ (22-30); Dem: 6,02 $\mu \mathrm{m}$ (5-8); Ex: 1,06 $\mu \mathrm{m}$; Orn: < $1 \mu \mathrm{m}$.

Observaçóes ecológicas: Herbácea, com ocorrência na Amazônia, caatinga, cerrado, mata atlântica, pampa e pantanal, popularmente conhecida como falso alecrim (FLORA DO BRASIL, 2018; ARRUDA, 2009).

\section{Kyllinga brevifolia Rottb. (Figura $3 \mathrm{M}-\mathrm{N}$ )}

Descrição polínica: Mônade, pequeno a média, simetria bilateral, anisopolar, âmbito subcircular, prolato-esferoidal (em forma de pêra, com maior tamanho em um dos pólos, apresentado em DEM e Dem), inaperturado, apresenta 3 a 5 áreas de fragilidade na exina, exina microrreticulada. Sexina e nexina de mesma espessura. P: 24,8 $\mu \mathrm{m}$ (22-29); DEM: 22,42 $\mu \mathrm{m}$ (19,5-25); Dem: 4,1 $\mu \mathrm{m}$ (5-7); Ex: 1,06 $\mu \mathrm{m}$; Orn: < $1 \mu \mathrm{m}$.

Observaçóes ecológicas: Herbácea, com ocorrência no Rio Grande do Sul, em todas as regióes fisiográficas, geralmente associada a ambientes úmidos e sombreados, popularmente conhecida como falso junquinho (TREVISAN et al., 2007).

\section{Poaceae}

Chascolytrum calotheca (Trin.) Essi, Longhi-Wagner \& Souza-Chies (Figura 3 O)

Descrição polínica: Mônade, médio, simetria radial, heteropolar, âmbito circular, esférico, monoporado, poro com ânulo com aproximadamente $3 \mu \mathrm{m}$ de diâmetro, ânulo com $7 \mu \mathrm{m}$ de diâmetro, exina microequinada. Sexina e nexina de mesma espessura. D: 26,88 $\mu \mathrm{m}$ (20-29); Ex: $1 \mu \mathrm{m}$; Or: $<1 \mu \mathrm{m}$. 
Observaçóes ecológicas: Herbácea, ocorrendo no cerrado, mata atlântica e pampa, popularmente conhecida como treme-treme (FLORA DO BRASIL, 2018)

\section{Melica brasiliana Ard. (Figura 3 P)}

Descrição polínica: Mônade, médio, simetria radial, heteropolar, âmbito circular, esférico, monoporado, poro com ânulo com aproximadamente $4 \mu \mathrm{m}$ de diâmetro,ânulo com $9 \mu \mathrm{m}$ de diâmetro, exina microequinada. Sexina e nexina de mesma espessura. D:

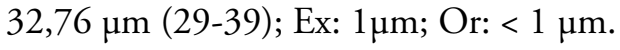

Observaçóes ecológicas: Herbácea, com ocorrência no Rio Grande do Sul, em todas as regióes fisiográficas, popularmente conhecida como guizo-de-cascavel (LONGHIWAGNER, 1987)

\section{Rosaceae}

Potentilla sp. L. (Figura 3 Q-R)

Descrição polínica: Mônade, pequeno, simetria radial, isopolar, âmbito subtriângular, subprolato, tricolporado, colpo longo, endoabertura lalongada com presença de fastígio, exina estriado-microrreticulada. Sexina e nexina de mesma espessura. P: 20,68 $\mu \mathrm{m}$ (16-24); E: 17,46 $\mu \mathrm{m}$ (13-23); Ex: 1,12 $\mu \mathrm{m}$.

Referência prévia: García e Sánchez, 2004.

Observaçóes ecológicas: Herbácea, com ocorrência na mata atlântica (FLORA DO BRASIL, 2018)

\section{Begoniaceae}

Begonia sp. C. Agardh (Figura 3 S-T)

Descrição polínica: Mônade, pequeno, simetria radial, isopolar, âmbito subtriangular, perprolato, tricolporado, colpo longo com margo e constricto na regiáo equatorial, endoabertura lalongada, exina estriada. Sexina e nexina de mesma espessura. P: 20,56 $\mu \mathrm{m}$ (19-22); E: 7,92 $\mu \mathrm{m}$ (6-10); Ex: 1,02 $\mu \mathrm{m}$.

Referência prévia: Evaldt et al., 2009, Bauermann et al., 2013.

Observaçóes ecológicas: Arbusto, com ocorrência na Amazônia, caatinga, cerrado e mata atlântica (FLORA DO BRASIL, 2018)

\section{Myrtaceae}

Myrrhinium atropurpureum Schott (Figura 4 A)

Descrição polínica: Mônade, pequeno, simetria radial, isopolar, âmbito triângular, peroblato, sincolporado, colpo longo formando triângulo no apocolpo, endoabertura circular, exina escabrada. Sexina e nexina de mesma espessura. P: 5,3 $\mu \mathrm{m}$ (4-7); E: 17,18 $\mu \mathrm{m}$ (14-21); Ex: $1 \mu \mathrm{m}$; Orn: < $1 \mu \mathrm{m}$.

Referência prévia: Evaldt et al., 2009. 
Observaçóes ecológicas: Árvore, com ocorrência em todas as formaçôes florestais do Rio Grande do Sul, popularmente conhecida como carrapato, pau-ferro (SOBRAL et al., 2006).

\section{Melastomataceae}

Leandra sp. Raddi (Figura 4 B-C)

Descrição polínica: Mônade, pequeno, simetria radial, isopolar, âmbito circular, prolato, tricolporado, heterocolpado, três colpos longos com endoabertura circular e três pseudocolpos, exina microrreticulada. Sexina e nexina de mesma espessura. P: 14,84 $\mu \mathrm{m}$

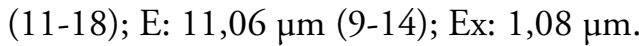

Referência prévia: Barth e Barbosa, 1975.

Observaçóes ecológicas: Arbusto, com ocorrência no Rio Grande do Sul, na floresta do alto Uruguai, floresta atlântica e floresta da encosta meridional da serra geral, atingindo eventualmente a depressão Central, popularmente conhecida como pixirica (SOBRAL et al., 2006).

\section{Miconia cabucu Hoehne (Figura 4 D-E)}

Descrição polínica: Mônade, pequeno, simetria radial, isopolar, âmbito circular, prolato, tricolporado, heterocolpado, três colpos longos com endoabertura circular e três pseudocolpos, exina psilada. Sexina e nexina de mesma espessura. P: 17,52 $\mu \mathrm{m}$ (16-20); E: $13 \mu \mathrm{m}(12-14)$; Ex: $1 \mu \mathrm{m}$.

Referência prévia: Barth e Barbosa, 1975.

Observaçóes ecológicas: Árvore, com ocorrência na floresta atlântica, popularmente conhecida como pixiricáo (FLORA DO RIO GRANDE DO SUL, 2018; FLORA DO BRASIL, 2018).

\section{Malvaceae}

Sida spinosa L. (Figura $4 \mathrm{~F}$ )

Descrição polínica: Mônade, grande, simetria radial, apolar, âmbito circular, esférico, pantoporado, exina equinada, Sexina mais espessa que a nexina. D: $68 \mu \mathrm{m}$ (57-79); Ex: 3,86 $\mu \mathrm{m}$; Orn: $5,7 \mu \mathrm{m}(5,7)$.

Referência prévia: Rede de Catálogos Polínicos online, 2017.

Observaçóes ecológicas: Subarbusto, com ocorrência na caatinga, cerrado, mata atlântica, popularmente conhecida como guanxuma de espinho (FLORA DO BRASIL, 2018; RCPOL, 2018)

\section{Rubiaceae}

Borreria palustris (Cham. \& Schltdl.) Bacigalupo \& E. L. Cabral (Figura 4 G-H)

Descrição polínica: Mônade, médio, simetria radial, isopolar, âmbito circular, subprolato, 11-colporado, colpo longo, endoabertura lalongada, exina microequinada. 
Espinhos de ápices afilados. Sexina e nexina de mesma espessura. P: 37,9 $\mu \mathrm{m}$ (31-46); E: 28,64 $\mu \mathrm{m}$ (23-34); Ex: 1,68 $\mu \mathrm{m}$.

Observaçóes ecológicas: Herbácea, com ocorrência na caatinga, cerrado e mata atlântica, popularmente conhecida como Poaia-do-brejo, erva-de-lagarto (FLORA DO BRASIL, 2018; FLORA DE SANTA CATARINA, 2018).

Galianthe brasiliensis (Spreng.) E. L. Cabral \& Bacigalupo (Figura 4 I-J)

Descrição polínica: Mônade, médio, simetria radial, isopolar, âmbito circular, oblatoesferoidal, 6-(7)-colporado, colpo curto, endoabertura lalongada, exina microrreticulada.

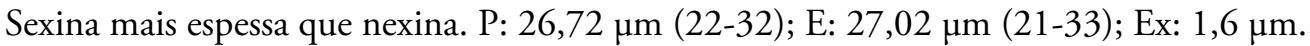

Referência prévia: Pire, 1997.

Observaçóes ecológicas: Arbusto, com ocorrência na caatinga, cerrado e mata atlântica, popularmente conhecida como grinalda-de-noiva (FLORA DO BRASIL, 2018; FLORA DE SANTA CATARINA, 2018).

\section{Solanaceae}

Solanum guaraniticum A. St. - Hil. (Figura 4 K-L)

Descrição polínica: Mônade, pequeno, simetria radial, isopolar, âmbito subtriângular, subprolato, tricolporado, colpo longo, endoabertura lalongada, exina microrreticulada. Sexina e nexina de mesma espessa. P: 25,24 $\mu \mathrm{m}$ (21-32); E: 19,56 $\mu \mathrm{m}$ (17-26); Ex: 1,1 $\mu \mathrm{m}$.

Observaçóes ecológicas: Arbusto, com ocorrência no Rio Grande do Sul, em todas as formaçóes vegetais, apresentando também comportamento ruderal, popularmente conhecida como jurubeba, falsa-jurubeba (MENTZ E OLIVEIRA, 2004; FLORA DO RIO GRANDE DO SUL, 2018).

\section{Solanum pseudocapsicum L. (Figura $4 \mathrm{M}-\mathrm{N}$ )}

Descrição polínica: Mônade, pequeno, simetria radial, isopolar, âmbito subtriângular, prolato-esferoidal, tricolporado, colpo longo, endoabertura lalongada com presença de fastígio, exina psilada. Sexina e nexina de mesma espessura. P: 15,42 $\mu \mathrm{m}$ (14-19); E: 15,24 $\mu \mathrm{m}$ (12-17); Ex: 1,14 $\mu \mathrm{m}$.

Referência prévia: Radaeski et al. 2014;Anil Kumar et al.,2015.

Observaçóes ecológicas: Subarbusto, com ocorrência no cerrado e mata atlântica, popularmente conhecida como peloteira (FLORA DO RIO GRANDE DO SUL, 2018; FLORA DO BRASIL, 2018).

\section{Solanum sanctae-catharinae Dunal (Figura 4 O-P)}

Descrição polínica: Mônade, pequeno, simetria radial, isopolar, âmbito subtriângular, prolato-esferoidal, tricolporado, colpo longo, endoabertura lalongada, exina microrreticulada. Sexina e nexina de mesma espessura. P: 12,62 $\mu \mathrm{m}$ (10-13); E: 11,38 $\mu \mathrm{m}$ (10-13); Ex: $1 \mu \mathrm{m}$.

Referência prévia: Barth e Duarte, 2008; Garralla e Cuadrado, 1998. 
Observaçóes ecológicas: Árvore, com ocorrência no Rio Grande do Sul, em todas as formaçôes florestais, popularmente conhecida como joá-manso e canena (SOBRAL et al., 2006).

\section{Asteraceae}

Austroeupatorium inulaefolium (Kunth) R. M. King \& H. Rob. (Figura 5 A-B)

Descrição polínica: Mônade, pequeno, simetria radial, isopolar, âmbito subtriangular, subprolato, tricolporado, colpo longo, endoabertura lalongada, exina equinada com presença de cava. Sexina e nexina de mesma espessura. P: 18,48 $\mu \mathrm{m}$ (15,7-20,73); E: 14,87 $\mu \mathrm{m}(10,82-18,69)$; Ex: $1 \mu \mathrm{m}$; Orn: 1,35 $\mu \mathrm{m}(0,77-1,88)$.

Referência prévia: Tellería, 2017.

Observaçóes ecológicas: Arbusto, com ocorrência em áreas de campo, vassoural e borda de mata, popularmente conhecida como cambará (BARÃO, 2016).

\section{Aspilia montevidensis (Spreng.) Kuntze (Figura 5 C-D)}

Descrição polínica: Mônade, médio, simetria radial, isopolar, âmbito subtriângular, oblato-esferoidal, tricolporado, colpo longo, endoabertura lalongada, exina equinada com presença de cava. Sexinae nexina de mesma espessura. Cerca de 15 espinhos em VP. P: 31 $\mu \mathrm{m}$ (27-36); E: 33,02 $\mu \mathrm{m}$ (28,5-38); Ex: 1,22 $\mu \mathrm{m}$; Orn: 4,54 $\mu \mathrm{m}(3,5)$.

Referência prévia: Evaldt et al., 2009; Radaeski et al., 2011; Radaeski et al., 2014.

Observaçóes ecológicas: Herbácea, no Rio Grande do Sul ocorre em todas as regióes fisiográficas; habita a vegetação campestre, campos rupestres, barrancos, banhados, margens de rios, beira de matas, ambientes ruderais, popularmente conhecida como margarida, malme-quer, mal-me-quer-amarelo e insulina (MONDIN, 2004).

\section{Baccharis articulata (Lam.) Pers. (Figura 5 E-F)}

Descrição polínica: Mônade, pequeno, simetria radial, isopolar, âmbito subtriângular, oblato-esferoidal, tricolporado, colpo longo, endoabertura lalongada, exina equinada com presença de cava. Sexina e nexina de mesma espessura. P: 20,08 $\mu \mathrm{m}$ (18-23); E: 21,96 $\mu \mathrm{m}$ (19-24); Ex: 1,18 $\mu$ m; Orn: 2,8 $\mu \mathrm{m}(2-3,5)$.

Referência prévia: Evaldt et al., 2009.

Observaçóes ecológicas: Subarbusto, com ocorrência no Rio Grande do Sul, em todas as regióes fisiográficas, popularmente conhecida como carqueja-doce, carqueja-miúda e carquejinha (HEIDEN et al., 2007).

\section{Chaptalia sp.Vent. (Figura 5 G-H)}

Descrição polínica: Mônade, médio, simetria radial, isopolar, âmbito subtriangular, oblato-esferoidal, tricolporado, colpo longo, endoabertura lalongada, exina equinada com presença de cava estreita. Sexina e nexina de mesma espessura. P: 24,44 $\mu \mathrm{m}$ (23-28); E: 25,3 $\mu \mathrm{m}$ (24-28); Ex: 1,02 $\mu \mathrm{m}$; Orn: 3,08 $\mu \mathrm{m}$ (2,5-4).

Referência prévia: Galvão et al., 2009; Cancelli,2008. 
Observaçóes ecológicas: Herbácea, com ocorrência na Amazônia, caatinga, cerrado, mata atlântica e pampa (FLORA DO BRASIL, 2018).

\section{Galinsoga parviflora Cav. (Figura $5 \mathrm{I}-\mathrm{J}$ )}

Descrição polínica: Mônade, médio, simetria radial, isopolar, âmbito subtriângular, prolato-esferoidal, tricolporado, colpo longo, endoabertura lalongada, exina equinada. Sexina e nexina de mesma espessura. P: 31,48 $\mu \mathrm{m}$ (28-36); E: 29,84 $\mu \mathrm{m}$ (27-34); Ex: 1,88 $\mu \mathrm{m}$; Orn: 4,36 $\mu \mathrm{m}$ (4-5). 2016.

Referência prévia: Cancelli et al., 2007; Evaldt et al., 2009; Stanski, Nogueira e Luz,

Observaçóes ecológicas: Herbácea, com ocorrência no Rio Grande do Sul, nas regióes fisiográficas do alto Uruguai, planalto médio, encosta inferior do nordeste, encosta superior do nordeste, missóes, litoral, depressão central e encosta do sudeste; habitando ambientes perturbados e áreas agrícolas, popularmente conhecida como fazendeiro e picãobranco (MONDIN, 2004).

\section{Jaegeria hirta (Lag.) Less. (Figura 5 K-L)}

Descrição polínica: Mônade, pequeno, simetria radial, isopolar, âmbito subtriângular, oblato-esferoidal, tricolporado, colpo longo, endoabertura lalongada, exina equinada com presença de cava estreita. Sexina e nexina de mesma espessura. P: 25,38 $\mu \mathrm{m}$ (24-28); E:

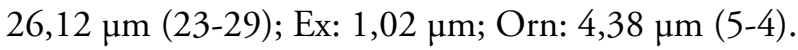

Referência prévia: Evaldt et al., 2009.

Observaçóes ecológicas: Herbácea, com ocorrência no Rio Grande do Sul, em todas as regiões fisiográficas do Estado; habitando preferencialmente locais úmidos e semisombreados das margens e interior de florestas, ruderal em beiras de estradas, capoeiras e áreas agrícolas, popularmente conhecida como botão-de-ouro (MONDIN, 2004).

\section{Senecio brasiliensis (Spreng.) Less. (Figura 5 M-N)}

Descrição polínica: Mônade, médio, simetria radial, isopolar, âmbito subtriângular, esférico, tricolporado, colpo longo, endoabertura lalongada, exina equinada com presença de cava estreita. Sexina e nexina de mesma espessura. P: $34 \mu \mathrm{m}$ (27-39); E: 33,94 $\mu \mathrm{m}$ (2937); Ex: 1,96 $\mu \mathrm{m}$; Orn: 2,8 $\mu \mathrm{m}(2-3,5)$.

Referência prévia: Stanski, Nogueira e Luz, 2016.

Observaçóes ecológicas: Herbácea, com ocorrência no Rio Grande do Sul, em todas as regióes fisiográficas, popularmente conhecida como maria-mole e mal-me-quer. (MATZENBACHER, 1998).

\section{Trichocline catharinensis Cabrera (Figura 5 O-P)}

Descrição polínica: Mônade, grande, simetria radial, isopolar, âmbito subtriangular, prolato, tricolporado, colpo longo, endoabertura lalongada, exina microequinada. Ectosexina com o dobro da espessura na região polar e endosexina mais espessa na regiâo equatorial. P: 70,06 $\mu \mathrm{m}$ (58-87); E: 45,18 $\mu \mathrm{m}$ (36-53); Ex: 9,94 $\mu \mathrm{m}$; Orn: < $1 \mu \mathrm{m}$. 
Referência prévia: Cancelli et al., 2007, 2010.

Observaçóes ecológicas: Herbácea, com ocorrência em campos de altitude e campo limpo, popularmente conhecido como cravo-do-campo (FLORA DO RIO GRANDE DO SUL, 2018; FLORA DO BRASIL, 2018).

Tabela 1: Informaçóes sobre a forma de vida, origem e tipo de vegetação dos táxons estudados

\begin{tabular}{|c|c|c|c|c|}
\hline Família & Espécie & Forma de vida & Origem & Ambiente \\
\hline Piperaceae & Piper mikanianum & Arbusto & Nativa & Campo/Floresta \\
\hline Iridaceae & Sisyrinchium sp. & Erva & Nativa & Campo \\
\hline Commelinaceae & Tripogandra sp. & Erva & Nativa & Campo/Floresta \\
\hline Bromeliaceae & Tillandsia sp. & Erva & Nativa & Campo/Floresta \\
\hline Cyperaceae & Cyperus surinamensis & Erva & Nativa & Campo Úmido \\
\hline Cyperaceae & Fimbristylis autumnalis & Erva & Nativa & Campo \\
\hline Cyperaceae & Kyllinga brevifolia & Erva & Nativa & Campo \\
\hline Poaceae & Chascolytrum calotheca & Erva & Nativa & Campo \\
\hline Poaceae & Melica brasiliana & Erva & Nativa & Campo \\
\hline Rosaceae & Potentilla sp. & Erva & Naturalizada & Campo \\
\hline Begoniaceae & Begonia sp. & Erva & Nativa & Campo/Floresta \\
\hline Myrtaceae & Myrrhinium atropurpureum & Arbusto/Árvore & Nativa & Floresta \\
\hline Melastomataceae & Leandra sp. & $\begin{array}{c}\text { Erva/Arbusto/ } \\
\text { Árvore }\end{array}$ & Nativa & Campo/Floresta \\
\hline Melastomataceae & Miconia cabucu & Árvore & Nativa & Floresta \\
\hline Malvaceae & Sida spinosa & Erva & Nativa & Campo \\
\hline Rubiaceae & Borreria palustris & Erva & Nativa & Floresta \\
\hline Rubiaceae & Galianthe brasiliensis & Arbusto & Nativa & Campo/Floresta \\
\hline Solanaceae & Solanum guaraniticum & Arbusto & Nativa & Campo/Floresta \\
\hline Solanaceae & Solanum pseudocapsicum & Arbusto & Nativa & Campo/Floresta \\
\hline Solanaceae & Solanum sanctae-catharinae & Árvore & Nativa & Floresta \\
\hline Asteraceae & Aspilia montevidensis & Erva & Nativa & Campo \\
\hline Asteraceae & Austroeupatorium inulaefolium & Arbusto & Nativa & Campo/Floresta \\
\hline Asteraceae & Baccharis articulata & Arbusto & Nativa & Campo \\
\hline Asteraceae & Chaptalia sp. & Erva & Nativa & Campo/Floresta \\
\hline Asteraceae & Galinsoga parviflora & Erva & Naturalizada & Floresta \\
\hline Asteraceae & Jaegeria hirta & Erva & Nativa & Campo/Floresta \\
\hline Asteraceae & Senecio brasiliensis & Erva & Nativa & Campo/Floresta \\
\hline Asteraceae & Trichocline catharinensis & Erva & Nativa & Campo \\
\hline
\end{tabular}

Fonte: Autoria própria. 
Figura 3. Grãos de pólen de Piperaceae: Piper mikanianum, VP (A), VE (B); Iridaceae: Sisyrinchium sp., VP (C), VE(D); Commelinaceae: Tripogandra sp. VP (E), VE (F); Bromeliaceae: Tillandsia sp., VP (G), VE (H); Cyperaceae: Cyperus surinamensis, VP (I), VE (J); Fimbristylis autumnalis, VP (K), VE (L); Kyllinga brevifolia, VP (M), VE (N); Poaceae: Chascolytrum calotheca, VP (O); Melica brasiliana, VP (P); Rosaceae: Potentilla sp., VP (Q), VE (R); Begoniaceae: Begonia sp., VP (S), VE (T).
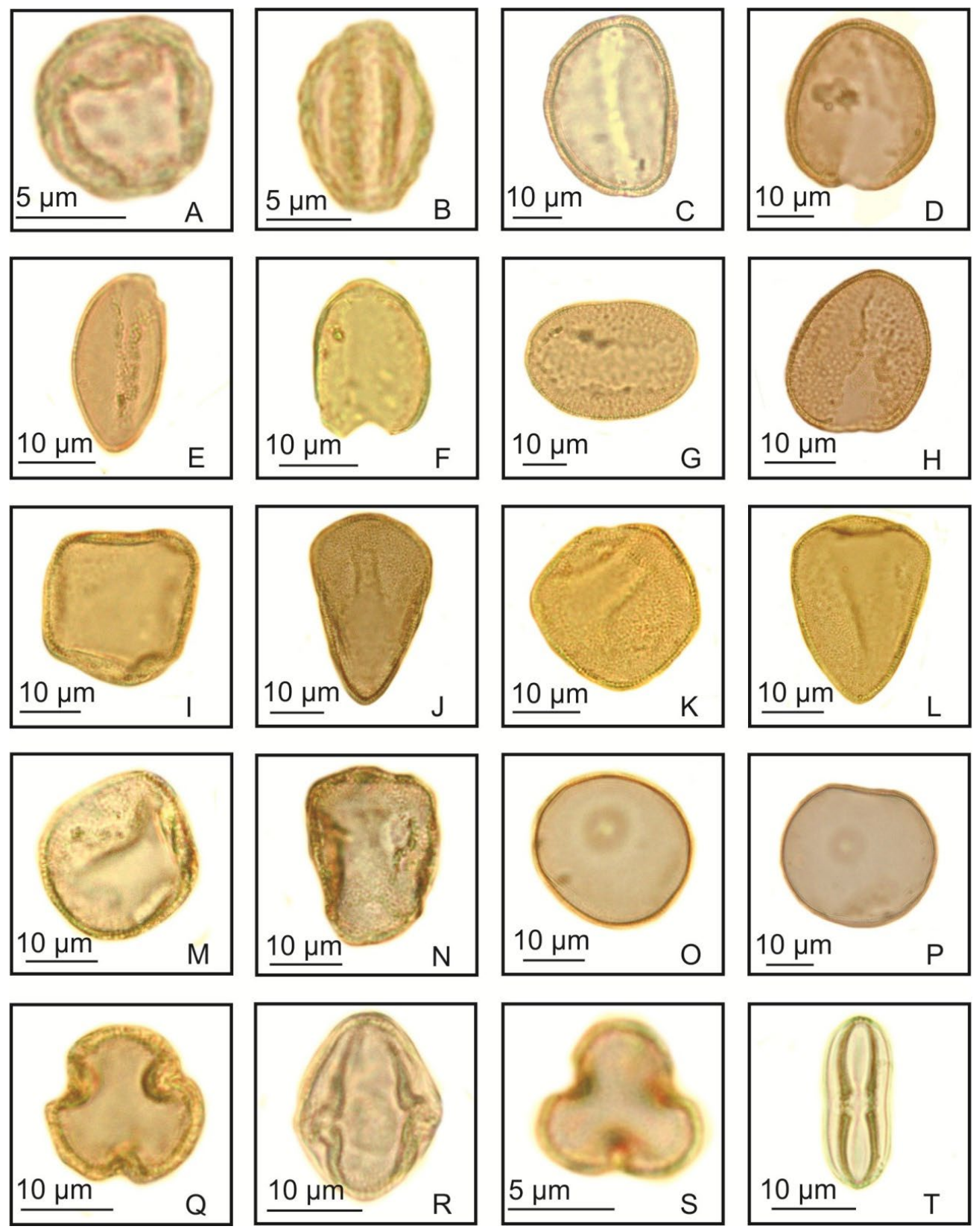

Fonte: Autoria própria. 
Figura 4. Grãos de pólen de Myrtaceae: Myrrhinium atropurpureum, (A); Melastomataceae: Leandra sp, VP (B), VE (C); Miconia cabucu, VP (D), VE (E); Malvaceae: Sida spinosa, (F); Rubiaceae: Borreria palustris, VP(G), VE (H); Galianthe brasiliensis, VP (I), VE (J); Solanaceae: Solanum guaraniticum, VP (K), VE (L); Solanum pseudocapsicum, VP (M), VE $(\mathrm{N})$; Solanum sanctae-catharinae VP $(\mathrm{O})$, VE $(\mathrm{P})$.
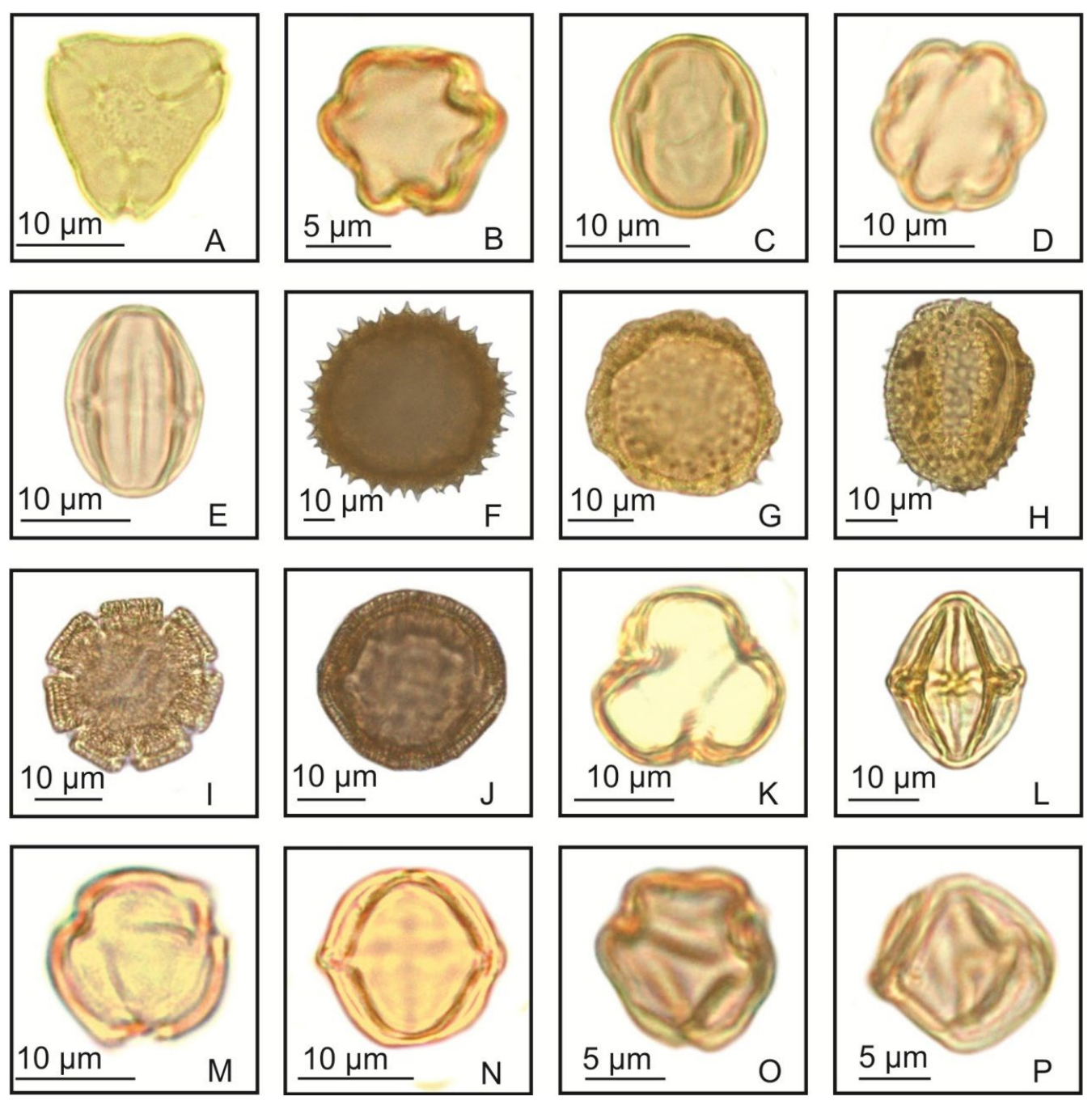

Fonte: Autoria própria. 
Figura 5. Grãos de pólen de Asteraceae: Austroeupatorium inulaefolium, VP (A), VE (B); Aspilia montevidensis, VP (C), VE (D); Baccharis articulata, VP (E), VE (F); Chaptalia sp., VP (G), VE (H); Galinsoga parviflora, VP (I), VE (J); Jaegeria hirta, VP (K), VE (L); Senecio brasiliensis, VP (M), VE (N); Trichocline catharinensis, VP (O), VE (P).
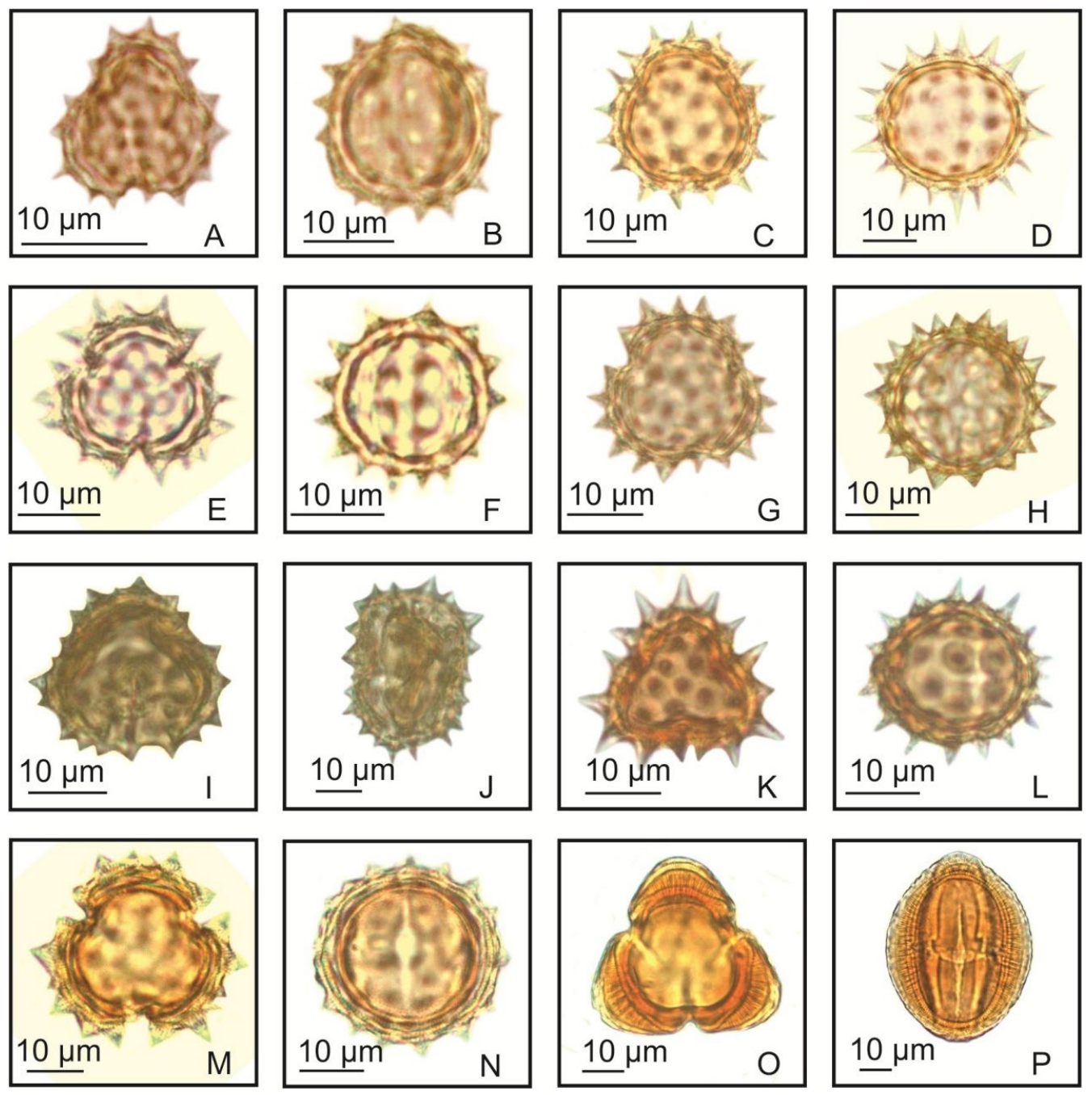

Fonte: Autoria própria.

Das 28 espécies descritas, 16 são inéditas para o RS (P. mikanianum, Tripogandra sp., C. surinamensis, F. autumnalis, K. brevifolia, C. calotheca, M. brasiliana, Potentilla sp., Leandra sp., M. cabucu, B. palustris, G. brasiliensis, S. guaraniticum, S. sanctae-catharinae, A. inulaefolium, S. brasiliensis).

Além disso, as descriçóes polínicas apresentadas de P. mikanianum, C. surinamensis, F. autumnalis, K. brevifolia, C. calotheca, M. brasiliana, B. palustris, S. guaraniticum eram desconhecidas em nível regional e global. 


\section{Campos}

A diversidade polínica das vegetaçóes campestres estudadas está representada principalmente por táxons das famílias Asteraceae (A. montevidensis, B. articulata e $T$. catharinensis) e Poaceae (C. calotheca e $M$. brasiliana). Dentre as espécies de Asteraceae, $A$. montevivensis, $B$. articulata e T. catharinensis não apresentaram diferenças morfológicas polinicas de seus tipos polinicos (tipo Heliantheae, tipo Baccharis e tipo Mutisia, respectivamente) anteriormente estabelecidos (CANCELLI et al., 2008; EVALDT et al., 2009; RADAESKI et al., 2014). Contudo, os grãos de pólen de A. montevidensis demonstraram tamanhos relativamente menores em relação ao pólen da mesma espécie descrito para outras regióes (CANCELLI et al., 2010; RADAESKI et al., 2014). Grãos de pólen de espécies de Cyperaceae não haviam sido descritos para o Rio Grande do Sul até o momento. K. brevifolia apresentou pólen inaperturado, enquanto Kyllinga elatior, descrita por Van Wichelen et al. (1999) apresenta um ulcus distal. Em relação aos grãos de pólen de Poaceae, C. calotheca e M. brasiliana demonstraram morfologias polínicas semelhantes ao pólen de Chascolytrum subaristatum e Melica sp. descrito para o Rio Grande do Sul (RADAESKI et al., 2016); o tamanho dos grãos de pólen destes táxons corresponde ao tipo polínico de gramíneas herbáceas campestres (RADAESKI et al., 2016). Potentilla sp. quando comparada a Potentilla candicans, Potentilla ehrenbergiana, Potentilla ranunculoides, Potentilla rivalis, Potentilla rubra, Potentilla richardii e Potentilla staminea, descritas por García e Sánchez (2004), mostrou diferença em tamanho, espessurada exina, ornamentação e tipo de endoabertura, logo presume-se que náo se trata de nenhuma dessas espécies. Sisyrinchium sp. apresentou características morfológicas semelhantes às descrições de Radaeski et al.(2011), exceto pelos diâmetros que se diferem, conclui-se que são espécies diferentes. S. spinosa mostrouse semelhante à descrição de Perveen et al. (1994), exceto pelo diâmetro do espinho, que houve uma pequena diferença, mas comparada com as descriçóes de Silva et al.(2014), os grãos se mostraram menores em diâmetro e espessura da exina.

\section{Floresta}

Os grãos de pólen estudados da vegetação florestal estão representados principalmente por Solanaceae (S. sanctae-catharinae), Myrtaceae (M. atropurpureum) e Melastomataceae (M. саbucu). $M$. atropurpureum não apresentou diferenças morfológicas anteriormente estabelecidas por Evaldt (2009), exceto pelo diâmetro equatorial, que obteve uma diferença de cerca de $10 \mu \mathrm{m}$ maior que a descrição anterior, e pela sexina ser menos espessa que a nexina. G. parviflora descrito por Evaldt (2009), apresentou grão de pólen com colpo médio, sexina mais espessa que nexina, não houve diferença em relação às descrições de Cancelli (2007). M. cabucu está de acordo com descriçóes de Barth e Barbosa (1975). B. palustris, comparada com Borreria brachystemonoides, grãos de pólen descritos por Bauermann et al. (2013), apresentou diferenças na quantidade e tipo de aberturas, tamanho e ornamentação de exina.

\section{Campo/Floresta}

A palinoflora que ocorre tanto em formaçóes florestais como campestres são representadas principalmente por táxons da família Asteraceae (A. inulaefolium, Chaptalia 
sp., J. hirta, S. brasiliensis). Begonia sp. comparada com as descrições do gênero Begonia de Evaldt et al. (2009) e Bauermann et al. (2013), se mostraram semelhantes morfologicamente. Para Evaldt (2009), J. hirta apresentou colpo médio, sexina mais espessa que nexina, diâmetros maiores e exina mais espessa. Tillandsia sp. apresentou descriçóes morfológicas semelhantes à Tillandsia duratii de Bauermann et al.(2013) e Radaeski et al.(2014), Tillandsia sp. descrita por Radaeski et al.(2014) apresentou maiores diâmetros. Chaptalia sp. apresentou considerável diferença de diâmetros e ornamentação de Chaptalia integerrima e Chaptalia nutans, descriçôes de Cancelli (2008) e Galvão (2009) respectivamente, as espécies apresentam diâmetros maiores e ornamentação microequinada, Chaptalia sp. não deve se tratar de Chaptalia integerrima ou Chaptalia nutans. Os grãos de pólen de P. mikanianum, comparada com Piper gaudichaudianum, não apresentam características relevantes para diferenciar as espécies. Tripogandra sp. apresenta características que não são semelhantes com as espécies descritas por Poole e Hunt (1980), entáo deve-se afirmar que se trata de outra espécie. As espécies do gênero Leandra descritas por Barth e Barbosa (1975) diferenciam-se principalmente quanto à ornamentação, não se encontrando nenhuma espécie microrreticulada, também há diferença nos diâmetros polar e equatorial,não deve ser nenhuma das espécies já descritas. G. brasiliensis, descrito por Pire (1997), não demonstrou diferenças relevantes entre as descriçóes. S. guaraniticum comparado a S. pseudocapsicum, apresentam características morfológicas que as diferem. Em relação à $S$. sanctae-catharinae, os grãos de pólen são semelhantes morfologicamente, se diferindo apenas pelos diâmetros. A. inulaefolium não obteve mudanças significativas no diâmetro polar para Tellería (2017), mas demonstrou uma diferença de $4,53 \mu \mathrm{m}$ de diâmetro equatorial. S. brasiliensis teve uma diferença de 7,1 $\mu \mathrm{m}$ da média do diâmetro polar, 7,34 $\mu \mathrm{m}$ do diâmetro equatorial, comparada à descrição de Stanski, Nogueira e Luz (2016).

\section{Campo úmido}

C. surinamensis apresenta diferenças em relação a espécies do gênero Cyperus descritas por Carreira et al. (2013) e Fernández (1987) por ter diâmetros diferentes, inaperturados e microrreticulados, características diferentes das encontradas por Carreira et al. (2013), que descreveu grãos maiores, 1- porado e exina psilada, e por Fernández (1987) que observou grãos menores, pantoaperturados e exina perfurada nas espécies do gênero Cyperus.

\section{Plantas exóticas}

G. parviflora (Asteraceae), e Potentilla sp. (Rosaceae) são exóticas na flora do estado. G. parviflora tem origem na América central.

\section{Conclusão}

O número alto de descrições polínicas inéditas (16) demonstra que a análise intensiva de formas modernas de grãos de pólen é efetiva para aprofundar o conhecimento sobre a diversidade polínica.

Os resultados obtidos neste trabalho promoveram uma melhor identificação taxonômica polínica para estudos paleopalinológicos que estão sendo desenvolvidos na região (SECCHI, 2017), ampliando o conhecimento sobre a sua ocorrência na vegetação pretérita. 
Os resultados das descrições polínicas indicaram a possibilidade de fazer identificação em nível de espécie nos táxons $K$. brevifolia, B. palustris, S. guaraniticum, S. sanctae-catharinae, C. surinamensis, devido as particularidades encontradas na morfologia polínica.

O conhecimento da flora polínica atual serve como apoio para promover açóes de reconstituição ambiental, definir a vegetação nativa e exótica, aliada a outras estratégias ecológicas, permite a recuperação das áreas degradas com mais garantia de sucesso, devido à utilização da flora natural já adaptada as condiçôes locais de clima, temperatura, relevo, etc.

\section{Agradecimentos}

Os autores agradecem aos pesquisadores da Universidade do Vale do Taquari Univates e ao Jorge Luiz Wolff pela identificação do material botânico. M. I. Secchi agradece a CAPES/PROSUP. Os autores também agradecem a ULBRA.

\section{Referências}

ADMINISTRAÇÃO MUNICIPAL DE ARVOREZINHA. Disponível em: <http://www. arvorezinhars.com.br/>. Acesso em 28 ago. 2017.

ALMEIDA JR, E. B.; PIMENTEL, R. M. M.; ZICKEL, C. S. Flora e formas de vida em uma área de restinga no litoral norte de Pernambuco, Brasil. Revista de Geografia, Recife, v. 24, n. 1, p. 19-34, 2008.

ANIL KUMAR, V. S.; NAIR, M.; MURUGAN, K. Pollen morphology of selected taxa of the genus Solanum from Southern Western Ghats, Kerala, India. Rheedea, Calicut, v. 25, n. 2, p. 128-145, 2015.

ARRUDA, R. et al. Composição e fenologia de espécies herbáceas nativas em reflorestamento heterogêneo. Floresta, Curitiba, v. 39, n. 3, p. 525-533, 2009.

APG - ANGIOSPERM PHYLOGENY GROUP. An update of the Angiosperm Phylogeny Group classification for the orders and families of flowering plants: APG IV. Botanical Journal of the Linnean Society, v. 181, n. 1, p. 1-20, 2016.

BARÃO, C. F. Levantamento de espécies da família Asteraceae no município de Sáo Gabriel, Rio Grande do Sul, Brasil. São Gabriel, Unipampa, 2016. 60 p.)

BARTH, O. M., BARBOSA, A. F. Catálogo sistemático dos pólens das plantas arbóreas do Brasil Meridional. XIX. Melastomataceae. Memórias do Instituto Oswaldo Cruz, v. 19, p. 39-63, 1975.

BARTH, O. M.; DUARTE, S. G. Pollen morphology of arboreal species of Solanaceae in the state of Santa Catarina, Brazil. Hoehnea, São Paulo, v. 35, n. 3, p. 379-386, 2008. 
BARTH, O. M.; MELHEM, T. S. Glossário ilustrado de palinologia. Campinas: Unicamp, 1988. 77 p.

BAUERMANN, S.G. et al. Pólen nas angiospermas: diversidade e evolução. Canoas: Ed. ULBRA, 2013. 216 p.

CANCELLI, R. R. Palinologia de Asteraceae: morfologia polínica e suas implicaçóes nos registros do Quaternário do Rio Grande do Sul. Porto Alegre: UFRGS, 2008. $155 \mathrm{p}$.

CANCELli, R. R., BAUERMANN, S. G., EVALDT, A. C. P. Contribuição à morfologia polínica da família Asteraceae Martinov. no Rio Grande do Sul-Parte I. Pesquisas, Série Botânica, São Leopoldo, v. 58, p. 347-374, 2007.

CANCELLI, R. R. et al. Catálogo palinológico de táxons da família Asteraceae Martinov, no Rio Grande do Sul, Brasil. Iheringia, Série Botânica, Porto Alegre, v. 65, n. 2, p. 201 280, 2010.

CARREIRA, L. M. M. Caracterização morfopolínica de macrófitas aquáticas da FlonaCaxiuanã II Monocotiledôneas e Licófitas In: Lisboa, P.L.B., Ed., Caxiuanã: Paraíso conservado. Museu Paraense Emilio Goeldi, Belém, 311-322, 2013.

CEMIN, G.; PÉRICO, E.; REMPEL, C. Uso de sistemas de informação geográfica para análise da estrutura da paisagem do município de Arvorezinha, RS. In: SIMPÓSIO BRASILEIRO DE SENSORIAMENTO REMOTO, 12, 2005, Goiânia. Anais... São José dos Campos: INPE, 2005. p. 2113-2120.

ERDTMAN, G. Pollenmorphology and plant taxonomy - Angiosperms. Waltham: The Chronica Botanica Co. 1952. p.539.

EVALDT, A.C.P. et al. Grãos de pólen e esporos do Vale do Rio Caí, nordeste do Rio Grande do Sul, Brasil: descrições morfológicas e implicaçóes paleoecológicas. GaeaJournal of Geoscience, São Leopoldo, v. 5, n. 2, p. 86-106, 2009.

FLORA DIGITAL DO RIO GRANDE DO SUL E SANTA CATARINA. Disponível em: <www.ufrgs.br/fitoecologia/florars/index.php>. Acesso em 04 dez. 2018.

FLORA DE SANTA CATARINA. Disponível em: <https://sites.google.com/site/ biodiversidadecatarinense/>. Acesso em 04 dez. 2018.

GALVÃO, M. N. et al. Palinologia de espécies de Asteraceae de utilidade medicinal para a comunidade da Vila Dois Rios, Ilha Grande, Angra dos Reis, RJ, Brasil. Acta botanica brasilica, Belo Horizonte, v. 23, n. 1, p. 247-258, 2009. 
GARRALLA, S.; CUADRADO, G. Solanaceae del Nordeste Argentino: morfología polínica del género Solanum L. In: Comunicaciones Científicas y Tecnológicas, Secretaría de Ciencia y Técnica. 2001, Corrientes. Anais...Corrientes: UNNE, 2001.

GARCÍA, D.L.Q., SÁNCHEZ, M.L.A. Morfología de los granos de polen de la Tribu Potentilleae (Rosaceae) del Valle de México, México. Polibotánica, Cidade do México,n. 18 , p. 87-97, 2004.

HEIDEN, G.; IGANCI, J. R. V.; BOBROWSKI, V. L.; MACIAS, L. Biogeografia de Baccharis sect. Caulopterae DC. (Asteraceae) no Rio Grande do Sul. Rodriguesia, v. 58 p. 787-796, 2007.

IBGE-Instituto Brasileiro Geografia e Estatística. 2016. Disponível em: <http://www.ibge. gov.br/>. Acessoem 28 ago. 2017.

LISTA DE ESPÉCIES DA FLORA DO BRASIL. Disponível em: <http://floradobrasil. jbrj.gov.br/>. Acesso em 28 ago. 2017.

LONGHI-WAGNER, H. M. Flora Ilustrada do Rio Grande do Sul. Gramineae - tribo Poeae. Boletim do Instituto de Biociências, Porto Alegre, v. 41, p. 1-191, 1987.

MATZENBACHER, N. I. O complexo "Senecionoide” (Asteraceae-Senecioneae) no Rio Grande do Sul - Brasil. Tese de Doutorado. Porto Alegre: Universidade Federal do Rio Grande do Sul. 1998. 274p.

MENTZ, L. A.; OLIVEIRA, P. L. O gênero Solanum na região Sul do Brasil. Pesquisas, Sér. Botânica, São Leopoldo, v. 54, p. 1-327, 2004.

MOAR, N. T., WILMSHURST, J. M. A key to the pollen of New Zealand Cyperaceae. New Zealand Jounal of Botany, Wellington, v. 41, n. 2, p. 325-334. 2003.

MONDIN, C. A. Levantamento da Tribo Heliantheae Cass. (Asteraceae), Sensu stricto, no Rio Grande do Sul, Brasil. Tese de Doutorado. Porto Alegre: Universidade Federal do Rio Grande do Sul. 2004. 344 p.

PÉRICO, E., CEMIN, G. Caracterização da paisagem do município de Arvorezinha, RS, com ênfase na dinâmica dos fragmentos florestais, por meio de sistemas de informaçóes geográficas (SIGs). Scientia Forestalis, Piracicaba, n. 70, p. 09-21, 2006.

PERVEEN, A. et al. Pollen flora of Pakistan - 1.Malvaceae. Pakistan Journal of Botany, Karachi, v. 26, n, 2, p. 421-440, 1994.

PILOTTO, N. et al. Origem floral de méis de Tetragonisca angustula (Latreille, 1811) e Scaptotrigona bipunctata (Lepeletier, 1836) no vale do Taquari, Rio Grande do Sul, Brasil. In: XVI Fórum de Pesquisa ULBRA. 2016, Canoas. Anais...Canoas: ULBRA, 2016. 
PIRE, S. M. Género Galianthe subg. Ebelia (Rubiaceae: Spermacoceae): studio palinológico. Annals of the Missouri Botanical Garden, Saint Loius, v. 84, p. 878-887, 1997.

POOLE, M. M., HUNT, D. Pollen Morphology and the Taxonomy of the Commelinaceae: An Exploratory Survey: American Commelinaceae: VIII. Kew Bulletin, Richmond, v. 34, n. 4, p. 639-660, 1980.

PUNT, W. et al. Glossary of pollen and spore terminology. Review of Palaeobotany and Palynology.v. 143, p. 1-81, 2007.

RADAESKI, J.N, EVALDT, A. C. P., LIMA, G. L., BAUERMANN, S.G. Grãos de pólen das formações campestres sul-brasileiras. Revista de Iniciação Científica da Universidade Luterana do Brasil, Canoas, v. 9, p. 59-67, 2011.

RADAESKI, J.N, EVALDT, A.C.P., LIMA, G.L., BAUERMANN, S.G. Diversidade de grãos de pólen e esporos dos Campos do sul do Brasil: descrições morfológicas e implicaçóes paleoecológicas. Iheringia, SérieBotanica, Porto Alegre, v. 69, n. 1, p. 107 132, 2014.

RADAESKI, J. N, EVALDT, A. C. P., BAUERMANN, S.G. Morfologia polínica de espécies da família Asteraceae Martinov nos cerros da Campanha do Rio Grande do Sul, Brasil. Iheringia. Série Botânica.v. 71, n. 3, p. 357-366, 2016.

RADAESKI, J. N.; CUNHA, D. J.; BAUERMANN, S. G. Diporate Pollen Grains of Poaceae Species: High Pollen Resolution for Reconstruction of Grasslands Vegetation. Journal of Agricultural Research, Troy, v. 2, p. 1-11, 2017.

REDE DE CATÁLOGOS POLÍNICOS ONLINE. Disponível em: <http://chaves.rcpol. org.br/>. Acesso em 28 ago. 2017.

SALGADO-LABOURIAU, M. L. Critérios e técnicas para o Quaternário. São Paulo: Ed. Blücher, 2007. 387 p.

SECCHI, M. I. Evoluçáo ambiental durante o Quaternário Superior em Arvorezinha/ RS: um milênio de influência humana na alteraçáo floresta. Tese de doutorado. Lajeado: UNIVATES, 2017. 174 p.

SILVA, C. I. et al. Catálogo polínico das plantas usadas por abelhas no Campus da USP de Ribeirão Preto. Ribeirão Preto: Holos, 2014. 153 p.

SOBRAL, M. et al. Flora arbórea e arborescente do Rio Grande do Sul, Brasil. São Carlos, RiMA/Novo Ambiente, 2006. 350 p. 
STANSKI, C., NOGUEIRA, M. K. F. S., LUZ, C. F. P. Pollen morphology of species of Asteraceae with medicinal utility from the region of Campos Gerais, Ponta Grossa, Paraná State, Brazil. Hoehnea, São Paulo, v. 43, n. 3, p. 349-360, 2016.

TELLERÍA, M. C. Spines vs. microspines: an overview of the sculpture exine in selected basal and derived Asteraceae with focus on Asteroideae. Journal of Plant Research, Tóquio, p. 1-11, 2017.

TREVISAN, R.; LÜDTKE, R.; BOLDRINI, I. O gênero Kyllinga Rottb.(Cyperaceae) no Rio Grande do Sul, Brasil. Revista Brasileira de Biociências, Porto Alegre, v. 5, n. 2-3, p. 27-36, 2007.

VAN WICHELEN, J. et al.Comparison of different treatments for LM and SEM studies and systematic value of pollen grains in Cyperaceae. Grana, Stockholm, v.38, p. 50-58, 1999. 